\title{
Probing Neutrino Mass Hierarchy by the Charged-Current and Neutral-Current Events of Supernova Neutrinos in Scintillation Detectors
}

\author{
Kwang-Chang Lai* \\ Center for General Education, Chang Gung University, Kwei-Shan, Taoyuan, 333, Taiwan \\ E-mail: kcl@mail.cgu.edu.tw
}

\section{Fei-Fan Lee}

Institute of Physics, National Chiao Tung University, Hsinchu, 300, Taiwan

E-mail: fflee@mail.nctu.edu.tw

\section{Feng-Shiuh Lee}

Department of Electrophysics, National Chiao Tung University, Hsinchu, 300, Taiwan

E-mail: leefs.py94@nctu.edu.tw

\section{Guey-Lin Lin}

Institute of Physics, National Chiao Tung University, Hsinchu, 300, Taiwan

E-mail: glin@mail.nctu.edu.tw

\begin{abstract}
The neutrino mass hierarchy is one of the neutrino fundamental properties yet to be determined. We introduce a method to determine neutrino mass hierarchy by comparing the events of neutral current (NC) interactions, $v(\bar{v})+p \rightarrow v(\bar{v})+p$, and inverse beta decays (IBD), $\bar{v}_{e}+p \rightarrow n+e^{+}$, of supernova neutrinos from accretion and cooling phases in scintillation detectors. Neutrino flavor conversions inside the supernova are sensitive to neutrino mass hierarchy. Due to MikheyevSmirnov-Wolfenstein effects, the full swapping of $\bar{v}_{e}$ flux with the $\bar{v}_{x}(x=\mu, \tau)$ one occurs in the inverted hierarchy, while such a swapping does not occur in the normal hierarchy. As a result, the ratio of high energy IBD events to NC events for the inverted hierarchy is higher than the ratio for the normal hierarchy. Since the luminosity of $\bar{v}_{e}$ is larger than that of $v_{x}$ in accretion phase while the luminosity of $\bar{v}_{e}$ becomes smaller than that of $v_{x}$ in cooling phase, we calculate this ratio for both accretion and cooling phases. By analyzing the change of this event ratio from accretion phase to cooling phase, one can determine the neutrino mass hierarchy.
\end{abstract}

The European Physical Society Conference on High Energy Physics

5-12 July, 2017

Venice

${ }^{*}$ Speaker. 


\section{Introduction}

Neutrino mass hierarchy (NMH) is one of the unresolvable mysteries in neutrino physics. Among various proposals to identify NMH, intensive efforts have been devoted to studying neutrinos from galactic SNe. As these neutrinos propagate outward, they can experience significant flavor transitions before arriving at the terrestrial detectors. The flavor conversions caused by the well-known Mikheyev-Smirnov-Wolfenstein (MSW) effect [1,2] depends on the neutrino mass hierarchy. In addition, it has been suggested that, due to the large neutrino number density in the deep region of the core, coherent $v-v$ forward scatterings may lead to collective flavor conversion $v_{e} \bar{v}_{e} \leftrightarrow v_{x} \bar{v}_{x}(x=\mu, \tau)$ over the entire energy range. This collective effect on the flavor transition of SN neutrinos depends crucially on the neutrino mass hierarchy and may also leave imprints on the neutrino spectra.

Most of the methods that use SN neutrinos for determining the neutrino mass hierarchy are based on the interactions of these neutrinos with atomic nuclei and free protons. The major interaction channel for neutrino detection is the inverse beta decay (IBD), $\bar{v}_{e}+p \rightarrow n+e^{+}$. While the liquid scintillation detector is sensitive to $\bar{v}_{e}$, the liquid argon detector has a good sensitivity to $v_{e}$ via charged-current interactions ${ }^{40} \mathrm{Ar}+v_{e} \rightarrow{ }^{40} \mathrm{~K}^{*}+e^{-}$. The detection of other species of SN neutrinos was proposed by measuring the neutral-current (NC) interactions, $v+p \rightarrow v+p[3,4]$.

Inspired by the capability of detecting thousands of neutrino events from a galactic supernova with next-generation scintillation detectors, we proposed to identify the neutrino mass hierarchy by comparing IBD and NC interactions inside the scintillators [5]. In the previous work, a set of specific mean energies of different flavors and luminosity equipartition between flavors for SN neutrinos are adopted. Instead of a unique scenario, models with different sets of mean energies and partitions of luminosities are explored in this work and the evolution with time of SN neutrinos is also accounted for in calculating IBD and NC events in the scintillation detector. We study how $\mathrm{NMH}$ relates to the way the IBD and NC events change with time during a SN explosion.

\section{Supernova neutrino events in liquid scintillation detectors}

A SN emits a total energy $\mathscr{E} \approx 10^{53}$ erg over a burst $\Delta t \approx 10$ s in neutrinos of all six flavors. The neutrino flavors $v_{\mu}, v_{\tau}$ and their antiparticles have similar interactions and thus similar average energies and fluences. Therefore, the total energy is divided as $\mathscr{E}=\mathscr{E}_{v_{e}}+\mathscr{E}_{\bar{v}_{e}}+4 \mathscr{E}_{V_{x}}$. In this work, the condition of equipartition of energies and luminosities among the primary neutrino flavors, $\mathscr{E}_{v_{e}} \approx \mathscr{E}_{\bar{v}_{e}} \approx \mathscr{E}_{v_{x}}$ and $\mathscr{L}_{v_{e}} \approx \mathscr{L}_{\bar{v}_{e}} \approx \mathscr{L}_{v_{x}}$, is relaxed. The primary SN neutrino energy spectrum is typically not purely thermal. We adopt a Keil parametrization [6] for the neutrino fluence

$$
F_{\alpha}^{0}(E)=\frac{\Phi_{\alpha}}{<E_{\alpha}>} \frac{\left(1+\eta_{\alpha}\right)^{\left(1+\eta_{\alpha}\right)}}{\Gamma\left(1+\eta_{\alpha}\right)}\left(\frac{E}{<E_{\alpha}>}\right)^{\eta_{\alpha}} \exp \left[-\left(\eta_{\alpha}+1\right) \frac{E}{<E_{\alpha}>}\right],
$$

where $\Phi_{\alpha}=\mathscr{E}_{\alpha} /\left\langle E_{\alpha}>\right.$ is the time-integrated flux, $\left\langle E_{\alpha}\right\rangle$ is the average neutrino energy, and $\eta_{\alpha}$ denotes the pinching of the spectrum. In our calculation, we take $\eta_{\alpha}=3$ for all flavors and the mean energies are taken to be $<E_{v_{e}}>=12 \mathrm{MeV},<E_{\bar{v}_{e}}>=15 \mathrm{MeV}$, and $<E_{v_{x}}>=18 \mathrm{MeV}$ for all other flavors. If flavor conversions do not occur during the propagations of neutrinos from the $\mathrm{SN}$ 
core to the Earth, a SN at a distance $d$ thus yields a neutrino fluence

$$
F_{\alpha}=\frac{F_{\alpha}^{0}}{4 \pi d^{2}}=\frac{2.35 \times 10^{13}}{\mathrm{~cm}^{2} \mathrm{MeV}} \frac{\mathscr{E}_{\alpha}}{d^{2}} \frac{E^{3}}{<E_{\alpha}>^{5}} \exp \left(-\frac{4 E}{<E_{\alpha}>}\right)
$$

with $\mathscr{E}_{\alpha}$ in units of $10^{52} \mathrm{erg}, d$ in $10 \mathrm{kpc}$, and energies in $\mathrm{MeV}$.

As neutrinos propagate outwards from deep inside $\mathrm{SN}$ and finally reach the Earth, their flavor contents are modified by the MSW effect. The fluxes of $v_{e}$ and $\bar{v}_{e}$ arriving at the detector can be written as

$$
\begin{aligned}
& F_{e}=F_{x}^{0}, \\
& F_{\bar{e}}=\left(1-\bar{P}_{2 e}\right) F_{\bar{e}}^{0}+\bar{P}_{2 e} F_{\bar{x}}^{0},
\end{aligned}
$$

for the normal hierarchy, and

$$
\begin{aligned}
& F_{e}=P_{2 e} F_{e}^{0}+\left(1-P_{2 e}\right) F_{x}^{0}, \\
& F_{\bar{e}}=F_{\bar{x}}^{0},
\end{aligned}
$$

for the inverted hierarchy [7]. Here $P_{2 e}$ is the probability that a $v_{2}$ is observed as a $v_{e}$ and $P_{2 e}=$ $\sin ^{2} \theta_{12}$ is used in this work.
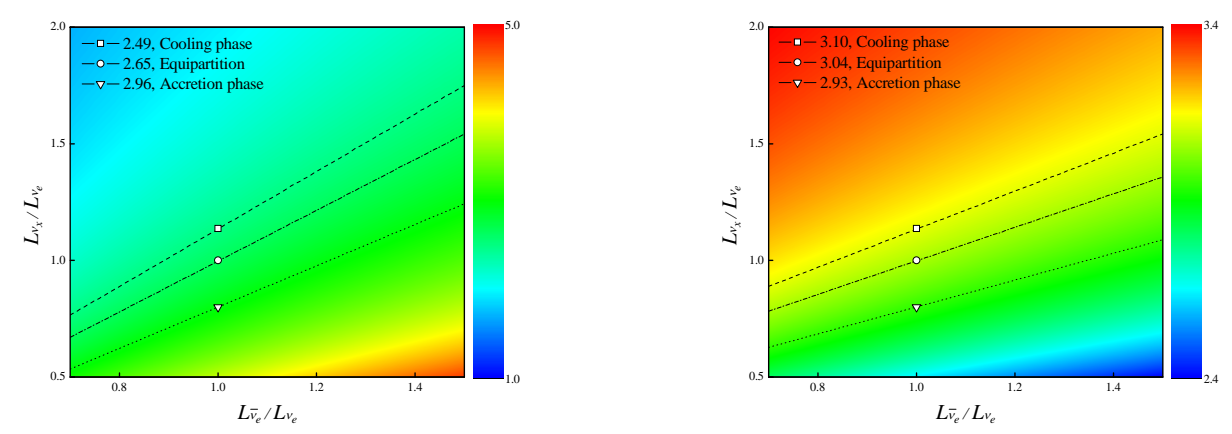

Figure 1: Ratios $R$ of IBD events to NC events for different energy compositions in $v_{e}, \bar{v}_{e}$, and $v_{x}$ for the normal hierarchy to the left and the inverted hierarchy to the right. $R$ values are scanned for $0.7<\mathscr{L}_{\bar{v}_{e}} / \mathscr{L}_{v_{e}}<$ 1.5 and $0.5<\mathscr{L}_{v_{x}} / \mathscr{L}_{v_{e}}<2.0$

Inside scintillation detectors, the most dominant interaction is the inverse beta decays (IBD), $\bar{v}_{e}+p \rightarrow n+e^{+}$. The neutrino-current $v$ p elastic scattering (NC), $v(\bar{v})+p \rightarrow v(\bar{v})+p$, can also yield comparable events. We define a ratio $R$ of total IBD events to total NC events,

$$
R \equiv \frac{N_{\mathrm{IBD}}}{N_{\mathrm{NC}}}
$$

and calculate the ratios for different SN neutrino scenarios. In this work, the condition of equipartition of energies and luminosities, $\mathscr{E}_{v_{e}} \approx \mathscr{E}_{\bar{v}_{e}} \approx \mathscr{E}_{v_{x}}$ and $\mathscr{L}_{v_{e}} \approx \mathscr{L}_{\bar{v}_{e}} \approx \mathscr{L}_{v_{x}}$, is relaxed. We then scan plausible ranges of luminosity ratios for $R$ values as shown in Fig. 1. It is shown that, for a given 


\begin{tabular}{lccc}
\hline \hline & Mark & $\mathscr{L}_{\bar{v}_{e}} / \mathscr{L}_{v_{e}}$ & $\mathscr{L}_{v_{x}} / \mathscr{L}_{v_{e}}$ \\
\hline Accretion & $\square$ & 1.00 & 0.80 \\
Equipartition & $\circ$ & 1.00 & 1.00 \\
Cooling & $\nabla$ & 1.00 & 1.14 \\
\hline
\end{tabular}

Table 1: NC events expected for various scintillation detectors. Chemical compositions and masses of scintillation materials, corresponding Birks constants $\left(k_{\mathrm{B}}\right)$, numbers of free protons $\left(N_{\mathrm{p}}\right)$, thresholds of proton recoil $(T)$, thresholds of minimum neutrino energy $\left(E_{v, \min }\right)$, and expected numbers of NC events for the detectors are listed here.

$\mathscr{L}_{\bar{v}_{e}} / \mathscr{L}_{v_{e}}$, the ratio $R$ decreases as $\mathscr{L}_{v_{x}} / \mathscr{L}_{v_{e}}$ increases for the normal hierarchy. On the contrary, the ratio $R$ increase as $\mathscr{L}_{v_{x}} / \mathscr{L}_{v_{e}}$ increases for the inverted hierarchy.

During the entire SN neutrino burst, neutrino emissions evolve with time as the SN explodes. An important feature of the evolution of SN neutrino emissions is that the hierarchy of luminosities in accretion and cooling phases are reversed. While $\mathscr{L}_{v_{e}} \approx \mathscr{L}_{\bar{v}_{e}}, \mathscr{L}_{v_{x}} / \mathscr{L}_{v_{e}}$ grows as SN neutrino emissions evolve from the accretion phase into the cooling phase and $\left(\mathscr{L}_{v_{x}} / \mathscr{L}_{v_{e}}\right)<1$ in the accretion phase and $\left(\mathscr{L}_{v_{x}} / \mathscr{L}_{v_{e}}\right)>1$ in the cooling phase. Therefore, we check three specific scenarios corresponding to the accretion phase, the equipartition model, and the cooling phase, respectively, as in Table 1. It is clear that, $R$ in the accretion phase will be smaller than $R$ in the cooling phase for the normal hierarchy, and this relation will be reversed for the inverted hierarchy.

\section{Conclusion}

We study the IBD and $v p$ elastic scattering. IBD and NC events in JUNO detector are explored for a few sets of mean energies of different neutrino flavors and for plausible ranges of luminosity ratios between flavors. Since NC events are blind to flavors, the ratio $R$ can be regarded as a measure of IBD events. As the SN neutrino emissions evolve from the accretion phase to the cooling phase, $\left(\mathscr{L}_{v_{x}} / \mathscr{L}_{v_{e}}\right)$ as well as $R$ change during the burst. From Fig. 1, we found that $R$ will decrease from the accretion phase to the cooling phase for the normal hierarchy and will increase for the inverted hierarchy. Therefore, the neutrino mass hierarchy can be determined by measuring IBD and NC events in scintillation detectors.

\section{References}

[1] L. Wolfenstein, Phys. Rev. D 17, 2369 (1978).

[2] S. P. Mikheev and A. Y. Smirnov, Sov. J. Nucl. Phys. 42, 913 (1985) [Yad. Fiz. 42, 1441 (1985)].

[3] J. F. Beacom, W. M. Farr and P. Vogel, Phys. Rev. D 66, 033001 (2002), arXiv:0205220.

[4] B. Dasgupta and J. F. Beacom, Phys. Rev. D 83, 113006(2011), arXiv:hep-ph/1103.2768.

[5] K.-C. Lai, F.-F. Lee, F.-S. Lee, G.-L. Lin, T.-C. Liu and Y. Yang, JCAP 1607, no. 07, 039 (2016), arXiv:1603.00692.

[6] M. T. Keil, G. G. Raffelt, and H.-T. Janka, Astrophys. J. 590, 971 (2003), arXiv:astro-ph/0208035.

[7] A. S. Dighe and A. Y. Smirnov, Phys. Rev. D 62, 033007 (2000), arXiv:hep-ph/9907423. 